\title{
ALMOST.PERIODICITY IN LINEAR TOPOLOGICAL SPACES AND APPLICATIONS TO ABSTRACT DIFFERENTIAL EQUATIONS
}

\section{GASTON MANDATA N'GUEREKATA}

\author{
Université de Bangui \\ Faculté des Sciences \\ BP 1450 Bangui \\ République Centrafricaine
}

(Received Apri1 28, 1982 and in revised form June 10, 1982)

ABSTKACT. Let $\mathrm{E}$ be a complete locally convex space (1.c.s.) and $\mathrm{f}: \mathrm{R} \rightarrow \mathrm{E}$ a continuous function; then $f$ is said to be almost-periodic (a.p.) if, for every neighbourhood (of the origin in E) $U$, there exists $\ell=\ell(U)>0$ such that every interval [a, at $\ell$ ] of the real line contains at least one point $\tau$ such that $f(t+\tau)-f(t) \varepsilon U$ for every $t \varepsilon R$. We prove in this paper many useful properties of a.p. functions in 1.c.s. and give Bochner's criteria in Fréchet spaces.

KEY WORUS ANV PHRASES. Almost-periodic junctions, Bochner's criteria, weakly almostperiodic junctions, abstract differential equations, perfect Frechet spaces, infinitesimal generator of equi-continous $C_{0}$-group. $19 \times 0$ maTiHEMATICS SUBJECT CLLASSIFICATIUN CODE. 34610.

1. INTRODUCTION

The notion of almost-periodic functions has been introduced by Bohl and Esclangon at the beginning of the century and widely studied by Bochner [1], [2] and many other mathematicians. The reader can see [3], [4], [5], [6], [7],... for what is written on the subject.

A definition of almost-periodic functions on a group and with values in a linear topological space is contained in the important 1935 paper of Bochner and Von Neumann [2]; we consider here the one suggested in [6] which is very easy to handle (see definition 1 below). liost of the results of Part I of this paper are known in Banach spaces. We give their extensions to linear topological spaces.

In Section 5 of our paper, we study almost-periodicity of solutions of some abstract differential equations of the form : $x^{\prime}(t)=A x(t)+f(t),-\infty<t<\infty$, in Fréchet spaces.

We suppose the reader is acquainted with elementary properties of linear topological spaces (see for example [8]).

We consider a locally convex space $E=E(\tau)$ over the field $\phi(\phi=R$ or $C)$; its topology $\tau$ is generated by a family of continous semi-norms $Q=\{p, q, \ldots\}$.

We assume $\mathrm{E}$ is a Hausdorff space. A basis of neighbourhoods (of the origin in E) contains sets of the form $U=U\left(\varepsilon ; p_{i}, 1 \leq i \leq n\right)=\left\{x \varepsilon k ; p_{i}(x)<\varepsilon, i=1, \ldots, n\right\} \quad p_{i} \varepsilon Q$. $E$ is called a Fréchet space if $\tau$ is induced by an invariant and complete metric. If 
$E$ is a Fréchet space, we may take $Q=\left\{p_{i}\right\}_{i=1}^{\infty}$. A subset $D \subseteq E$ is dense in $E$ if every $\mathrm{x} \varepsilon \mathrm{t}$ is the limit of a generalized sequence of elements of $\mathrm{D}$. A linear operator A : $D(A) \rightarrow E$ with domain $D(A)$ dense in $E$ is closed if its graph $G(A)$ is a closed subset of the product space $E \times E$.

THEOREM. (See [9]). Let $\mathrm{E}$ be a complete locally convex space. Then the linear operator $A: D(A) \rightarrow E$ is closed iff for every generalized sequence $\left(x_{\mu}\right)$ in $D(A)$ such that $\lim _{\mu} \mathrm{x}_{\mu}=\mathrm{x}$ and $\lim _{\mu} A \mathrm{x}_{\mu}=\mathrm{y}$ we have $\mathrm{x} \varepsilon \mathrm{D}(\mathrm{A})$ and $\mathrm{Ax}=\mathrm{y}$.

CUROLLARY. Every continous linear operator defined on all $\mathrm{E}$ is closed.

In a locally convex space $E$, a subset $X$ is called totally bounded if, for every neighbourhood (of the origin) $U$, there corresponds a finite set $Y$ such that $X \subset Y+U$. 2. ALIIOST PERIOdiC FUNCTIONS WITH VALUES IN A LOCALly CONVEX SPACE.

DEFINITION 1. Let $E$ be a complete locally convex space (l.c.s.). A continous function $f: R \rightarrow E$ is called almost-periodic (a.p.) if for each neighbourhood (of the origin) $U$, there exists a real number $\ell=\ell(U)>0$ such that every interval $[a, a+l]$ contains at least a point $\tau$ such that $f(t+\tau)-f(t) \varepsilon U$ for every $t \varepsilon R$.

Obviously $\tau=\tau_{U}$ and we call it a U-translation number of the function $f$. The following two theorems are known (see [6]). We give here a proof of the second one.

THEOREli 1. (a) If $\mathrm{f}: \mathrm{R} \rightarrow \mathrm{E}$ is $\mathrm{a} \cdot \mathrm{p}$. then $\mathrm{f}$ is uniformly continuous on $\mathrm{R}$. (b) If $\left(f_{n}\right)_{n=1}^{\infty}$ is a sequence of a.p. functions which converge uniformly on $R$ to a function $f$, then $f$ is also a.p..

THEOREM 2. If $\mathrm{f}$ is a.p., then $\{\mathrm{f}(\mathrm{t}) ; t \in \mathrm{R}\}$ is totally bounded in $E$.

PROOF. Let $U$ be a given neighbourhood, and $V$ a symmetric neighbourhood such that $V+V \subset U$; let $l=l(V)$ as in definition 1 . By continuity of $f$, the set $\{f(t)$; $t \varepsilon[0, \ell]\}$ is compact in $E$ (see [8] proposition 7, p. 53). But in a 1.c.s., every compact set is totally bounded (see [8] theorem 5, p. 60): therefore there exists $x_{1}, \ldots, x_{v}$ such that for every $t \varepsilon[0, \ell]$, we have $f(t) \varepsilon \underset{j=1}{U}\left(x_{j}+V\right)$.

Take an arbitrary $t \in R$ and consider $\tau \varepsilon[-t,-t+l]$ a $V$ - translation number of $f$. Then we have:

$$
f(t+\tau)-f(t) \varepsilon V .
$$

Choose $x_{k}$ between $x_{1}, \ldots, x_{v}$ such that

$$
f(t+\tau) \varepsilon x_{k}+v \text {. }
$$

Let us write $f(t)-x_{k}=[f(t)-f(t+\tau)]+\left[f(t+\tau)-x_{k}\right]$. Then by (2.1) and (2.2) we get $f(t)-x_{k} \varepsilon U$ and therefore $f(t) \varepsilon x_{k}+U$; as $t$ is arbitrary we conclude:

The theorem is proved.

$$
\{f(t) ; t \varepsilon R\} \subset \underset{j=1}{U}\left(x_{j}+U\right)
$$

REMARK 1. If $E$ is a Fréchet space, then $\{f(t): t \varepsilon R\}$ is relatively compact in $E$ if $f$ is a.p.. For in every complete metric space, relative compacity and totally boundedness are equivalent $([13], p .13)$. We then conclude every sequence $\left(f\left(t_{n}\right)\right)_{n=1}^{\infty}$ contains a convergent subsequence.

THEOREM 3. Let $\mathrm{E}$ be a complete 1.c.s. If $\mathrm{f}: \mathrm{R} \rightarrow \mathrm{E}$ is $\mathrm{a} \cdot \mathrm{p}$. then the functions $\lambda f(\lambda \varepsilon \Phi)$ and $\overline{\mathrm{f}}$ defined by $\overline{\mathrm{f}}(\mathrm{t}) \equiv \mathrm{f}(-t)$ are al so a.p.

PROOF. $\lambda \mathrm{f}$ is obviously a.p.. Let us consider $\overline{\mathrm{f}}$; by almost-periodicity of $\mathrm{f}$, if $U$ is a given neighbourhood, there exists $\ell=\ell(U)$ such that every interval [a,a+l] 
contains $\tau$ such that $f(t+\tau)-f(t) \varepsilon U$ for every $t \varepsilon R$. Put $s=-t$; we get :

$$
\overline{\mathrm{f}}(s-\tau)-\overline{\mathrm{f}}(\mathrm{s})=\mathrm{f}(-\mathrm{s}+\tau)-\mathrm{f}(-\mathrm{s})=\mathrm{f}(\mathrm{t}+\tau)-f(t) .
$$

Therefore $\bar{f}(s-\tau)-\bar{f}(s) \varepsilon U$ for every $s \varepsilon R$, which shows $f$ is a.p. with $-\tau$ as a U-translation number.

3. BOCHNER'S CRITERIA AND OTHER PROPERTIES.

We first give theorem 4 we prove as theorem 6.6 in [6].

THEOREM 4. Let $\mathrm{E}$ be a Fréchet space and $\mathrm{f}: \mathrm{R} \rightarrow \mathrm{E}$ a.p.; then for every real sequence $\left(s^{\prime}{ }_{n}\right)_{n=1}^{\infty}$, there exists a subsequence $\left(s_{n}\right)_{n=1}^{\infty}$ such that $\left(f\left(t+s_{n}\right)\right)_{n=1}^{\infty}$ is uniformly convergent in $t \varepsilon R$.

PROOF. Consider the sequence of functions $\left(f_{s_{n}}\right)_{n=1}^{\infty}$ corresponding to $\left(s_{n}\right)_{n=1}^{\infty}$ and let $S=\left(n_{n}\right)_{n=1}^{\infty}$ be a dense sequence in $R$. By remark 1 , we can extract from $\left(f\left(n_{1}+s_{n}\right)\right)_{n=1}^{\infty}$ a convergent subsequence, for $\{f(t) ; t \varepsilon R\}$ is relatively compact in $E$.

Let $\left(f_{s_{1}, n}\right)_{n=1}^{\infty}$ be the subsequence of $\left(f_{s_{n}}\right)_{n=1}^{\infty}$ which converges at $n_{1}$. We app]y the same argument as above to the sequence $\left(f_{s_{1, n}}\right)_{n=1}^{\infty}$ to choose a subsequence $\left(f_{s_{2}, n}\right)_{n=1}^{\infty}$ which converges at $n_{2}$. We continue the process and consider the diagonal sequence $\left(f_{s_{n, n}}\right)_{n=1}^{\infty}$ which converges for each $n_{n}$ in $S$. Call this last sequence by $\left(f_{r_{n}}\right)_{n=1}^{\infty}$. Now we are going to show it is uniformly convergent in $R$, i.e. for every neighbourhood $U$, there exists $N=N_{U}$ such that $f\left(t+r_{n}\right)-f\left(t+r_{m}\right) \varepsilon U$ for every $t \varepsilon R$ if $n, m>N$.

Consider an arbitrary neighbourhood $U$ and a symmetric neighbourhood $V$ such that $\mathrm{V}+\mathrm{V}+\mathrm{V}+\mathrm{V}+\mathrm{V} \subset \mathrm{U}$. Let $\ell=\ell(\mathrm{V})$ as in definition 1 . By uniform continuity of $f$ over $R$ (theorem 1), there exists $\delta=\delta_{V}>0$ such that

$$
f(t)-f\left(t^{\prime}\right) \varepsilon V
$$

for every $t, t^{\prime} \varepsilon R$ with $\left|t-t^{\prime}\right|<\delta$.

We divide the interval $[0, \ell]$ into $v$ subintervals of length smaller than $\delta$. Then, in each interval, we choose a point of $s$ and get $s_{0}=\left\{\xi_{1}, \ldots, \xi_{v}\right\}$. As $s_{0}$ is finite, $\left(f_{r_{n}}\right)_{n=1}^{\infty}$ is uniformly convergent over $s_{0}$; therefore there exists $\mathrm{N}=\mathrm{N}_{\mathrm{V}}$ such that

$$
f\left(\xi_{i}+r_{n}\right)-f\left(\xi_{i}+r_{m}\right) \varepsilon v
$$

for every $i=1, \ldots, v$ and if $n, m>N$.

Let $t \varepsilon R$ be arbitrary and $\tau \varepsilon[-t,-t+l]$ such that $f(t+\tau)-f(t) \in V$. Choose $\xi_{i}$ such that $\left|t+\tau-\xi_{i}\right|<\delta$; then $f\left(t+\tau+r_{n}\right)-f\left(\xi_{i}+r_{m}\right) \varepsilon V$, for every $n$. Therefore, if $\mathrm{n}, \mathrm{m}>\mathrm{N}$, we get:

$$
f\left(t+r_{n}\right)-f\left(t+r_{m}\right) \varepsilon U,
$$

which proves uniform convergence of $\left(f\left(t+r_{n}\right)\right)_{n=1}^{\infty}$.

To see (3.3) we write :

$$
\begin{aligned}
& f\left(t+r_{n}\right)-f\left(t+r_{m}\right)=\left[f\left(t+r_{n}\right)-f\left(t+r_{n}+\tau\right]\right. \\
& +\left[f\left(t+r_{n}+\tau\right)-f\left(\xi_{i}+r_{n}\right)\right]+\left[f\left(\xi_{i}+r_{n}\right)-f\left(\xi_{i}+r_{m}\right)\right] \\
& +\left[f\left(\xi_{i}+r_{m}\right)-f\left(t+r_{m}+\tau\right)\right] \\
& +\left[f\left(t+r_{m}+\tau\right)-f\left(t+r_{m}\right)\right],
\end{aligned}
$$

and we apply (3.2) or (3.3) to each term in square brackets. The theorem is proved.

We now state and prove Bochner's criteria :

THEOREM 5. Let $E$ be a Fréchet space. Then $f: R \rightarrow E$ is a.p. iff for every real 
sequence $\left(s_{n}^{\prime}\right)_{n=1}^{\infty}$ there exists a subsequence $\left(s_{n}\right)_{n=1}^{\infty}$ such that $\left(f\left(t+s_{n}\right)\right)_{n=1}^{\infty}$ converges uniformly in $t \in R$.

PROOF. The condition is obviously necessary by theorem 4; let us show it is sufficient; suppose $\mathrm{f}$ is not a.p.; then there exists a neighbourhood $U$ such that for every $\ell>0$, there exists an interval of length $\ell$ which contains no U-translation number of $\mathrm{f}$, or :

there exists an interval $[-a,-a+l]$ such that for every

$\tau \varepsilon[-a,-a+l]$ there exists $t=t_{\tau}$ such that $f(t+\tau)-f(t) \notin U$.

Let us consider $\tau_{1} \varepsilon R$ and an interval $\left(a_{1}-b_{1}\right)$ with $b_{1}-a_{1}>2\left|\tau_{1}\right|$ which contains no U-translation number of $f$. Now let $\tau_{2}=\frac{a_{1}-b_{1}}{2} ;$ then $\tau_{2} \tau_{1} \varepsilon\left(a_{1}, b_{1}\right)$ and therefore $\tau_{2}-\tau_{1}$ cannot be a U-translation number of $f$. Let us consider another interval $\left(a_{2}, b_{2}\right)$ with $b_{2}-a_{2}>2\left(j \tau_{1}|+| \tau_{2} \mid\right)$, which contains no $U$-translation number of $f$. Let $\tau_{3}=\frac{a_{2}-b_{2}}{2}$; then $\tau_{3}-\tau_{1}, \tau_{3}-\tau_{2} \quad \varepsilon\left(a_{2}, b_{2}\right)$ and therefore $\tau_{3}{ }^{-\tau_{1}}$ and $\tau_{3}-\tau_{2}$ cannot be $U$-translation number of $f$. We proceed and get a sequence $\left(\tau_{n}\right)_{n=1}^{\infty}$ such that no $\tau_{m}-\tau_{n}$ is a U-translation number of $f$;

$$
f\left(t+\tau_{m}-\tau_{n}\right)-f(t) \notin U \text {. }
$$

Put $\sigma=\sigma_{\mathrm{mn}}=t-\tau_{A}$; then (3.4) becomes:

$$
\mathrm{f}\left(\sigma+\tau_{\mathrm{m}}\right)-\mathrm{f}\left(\sigma+\tau_{\mathrm{n}}\right) \notin \mathrm{U} \text {. }
$$

Suppose there exists a subsequence $\left(\tau^{\prime}{ }_{n}\right)_{n=1}^{\infty}$ of $\left(\tau_{n}\right)_{n=1}^{\infty}$ such that $\left(f\left(t+\tau^{\prime}{ }_{n}\right)\right)_{n=1}^{\infty}$ converges uniformly in $t \varepsilon R$; then for every neighbourhood $V$, there exists $N=N_{V}$ such that if $m, n>N$ (we may take $m>n$ ), then we have:

$$
f\left(t+\tau_{m}^{\prime}\right)-f\left(t+\tau{ }_{n}^{\prime}\right) \varepsilon V
$$

for every $t \in R$.

But this contradicts (3.5); it suffices to take $U=V$ and $\sigma_{m n}=t_{m n}$ in (3). Therefore $\left(f\left(t+\tau_{n}\right)\right)_{n=1}^{\infty}$ does not contain any subsequence which converges uniformly in $t$. The theorem is proved.

REMARK 2. Here we do not use metrizability of $E$ in the proof of the sufficiency of the condition.

THEOREM 6. Let $\mathrm{E}$ be a Frechet space and consider the functions $\mathrm{f}, \mathrm{g}, \mathrm{f}_{1}, \mathrm{f}_{2}$ : $\mathrm{R} \rightarrow \mathrm{E}$; then we have:

a) $f+g$ is a.p. in $E$ if $f$ and $g$ are a.p. in $E$

b) $F=\left(f_{1}, f_{2}\right)$ is a.p. in the product space $E \times E$ if $f_{1}$ and $f_{2}$ are a.p. in $\mathrm{E}$.

PROOF. It is very easy to prove a) and b) by using Bochner's criteria; we omit it. The reader can see $[9]$.

COROLLARY 1. If $\mathrm{f}_{1}$ and $\mathrm{f}_{2}$ are a.p. in the Fréchet space $\mathrm{E}$, then for every neighbourhood $U, f_{1}$ and $f_{2}$ have common U-translation numbers.

PROOF. Let $U$ be a given neighbourhood in $E$; by theorem 6 , the function $f(t)=$ $\left(f_{1}(t), f_{2}(t)\right.$ ) is a.p.. Consider now $\tau$ a $U$ x U-translation number of $f$; then $f(t+\tau)-$ $f(t) \varepsilon U \times U$, for every $t \varepsilon R$ and therefore $f_{i}(t+\tau)-f_{i}(t) \varepsilon U, i=1,2$, for every $t \varepsilon R ; \tau$ is then a U-translation number of $\mathrm{f}_{1}$ and $\mathrm{f}_{2}$.

REMARK 3. Theorem $6, \mathrm{~b})$ and corollary 1 are true even for $n$ functions, $n \geq 2$. 
4. WEAKLY A.P. FUNCTIONS; INTEGRATION OF A.P. FUNCTIONS.

Let $E$ be a complete locally convex space.

DEFINITION. A function $\mathrm{f}: \mathrm{K} \rightarrow \mathrm{E}$ is called weakly a.p. (we write W.a.p.) in $\mathrm{E}$ if the numerical function $\left(x^{\star} f\right)(t)$ is a.p. for every $x^{\star} \varepsilon E$ where $E^{\star}$ is the dual space of E.

Obviously every a.p. function is w.a.p.; and if $f$ is w.a.p. then it is weakly continous and weakly bounded.

THEOREM 7. Let $\mathrm{E}$ be a complete 1.c.s. and $\mathrm{f}$ a w.a.p. and continous function; assume $\{F(t) ; t \in R\}$ is weakly bounded, where $F(t)=\int_{0}^{t} f(\sigma) d \sigma$; then $F(t)$ is w.a.p...

PROOF. We first note existence of the integral because of continuity of $f$ over R. Take any $x^{*} \in E^{*}$; then $\left(x^{*} f\right)(t)$ is a.p.. By continuity of $x^{*}$, we have $\left(x^{*} F\right)(t)=$ $\int_{0}^{t}\left(x^{*} f\right)(\sigma) d \sigma$, which is bounded by our assumption. Now $\left(x^{*} F\right)(t)$ is a.p. (see [6], theorem 6.20). The theorem is proved.

THEOREM 8. Let $\mathrm{E}$ be a Fréchet space and $\mathrm{f}: \mathrm{R} \rightarrow \mathrm{E}$ a given function; then $\mathrm{f}$ is a.p. if $f$ is w.a.p. and $\{f(t): t \varepsilon R\}$ is relatively compact in $E$.

PROOF. The condition is obviously necessary. Let us show it is sufficient by contradiction. Suppose there exists $t_{0}$ such that $f$ is discontinuous at $t_{0}$. Then we can find a neighbourhood $U$ and two sequences $\left(s^{\prime} n_{1}\right)_{n=1}^{\infty}$ and $\left(s^{\prime}{ }_{n}\right)_{n=1}^{\infty}$ such that $\lim _{n \rightarrow \infty} s^{\prime} n_{1}=0=\lim _{n \rightarrow \infty} s^{\prime} n_{2}$ and

$$
\mathrm{f}\left(\mathrm{t}_{0}+\mathrm{s}^{\prime} \mathrm{n}_{1}\right)-\mathrm{f}\left(\mathrm{t}_{0}+\mathrm{s}^{\prime} \mathrm{n}_{2}\right) \not \mathrm{U}
$$

for every $n \varepsilon N$. By relative compacity of $\{f(t) ; t \varepsilon R\}$ we can extract $\left(s_{n_{1}}\right)_{n_{n}=1}^{\infty}$ and $\left(s_{n_{2}}\right)_{n=1}^{\infty}$ from the respective first two sequences such that $\underset{n \neq \infty}{1} f\left(t_{0}+s_{n_{1}}\right)=a_{1} \varepsilon E$ and $\underset{n}{1} \underset{j m}{f} f\left(t_{0}+s_{n_{2}}\right)=a_{2} \varepsilon E$. Consequently, using (4.1), we get $a_{1}-a_{2} \varepsilon E$ and therefore by the Hahn-Banach theorem ([13], corollary 1, p. 108), there exists $x^{\star} \varepsilon E^{\star}$ such that $x^{*}\left(a_{1}-a_{2}\right) \neq 0$; hence

$$
x *\left(a_{1}\right) \neq x *\left(a_{2}\right) \text {. }
$$

By continuity of $x^{*}$, we have:

$$
x *\left(a_{1}\right)=\lim _{n \rightarrow \infty} x * f\left(t_{0}+s_{n_{1}}\right)=\lim _{n \rightarrow \infty} x * f\left(t_{0}+s_{n_{2}}\right)=x *\left(a_{2}\right)
$$

which contradicts (4.2); $\mathrm{f}$ is therefore continuous over $\mathrm{R}$.

We are now going to show almost-periodicity of $f$; but first of all, we state and prove:

LEMMA 1. Let $E$ be a Fréchet space and $\Phi: R \rightarrow E$ be a.p.. Let $\left(s_{n}\right)_{n=1}^{\infty}$ be a real sequence such that $\lim _{n \rightarrow \infty} \Phi\left(s_{n}+n_{k}\right)$ exists for each $k=1,2, \ldots$, where $\left(n_{k}\right)_{k=1}^{\infty}$ is dense in $R$. Then $\left(\Phi\left(t+s_{n}\right)\right)_{n=1}^{\infty}$ is uniformly convergent in $t \varepsilon R$.

PROOF. Suppose by contradiction $\left(\Phi\left(t+s_{n}\right)\right)_{n=1}^{\infty}$ is not uniformly convergent in $t$; then there exists a neighbourhood $U$ such that for every $N=1,2, \ldots$, there exists $\mathrm{n}_{\mathrm{N}}, \mathrm{m}_{\mathrm{N}} \quad \mathrm{N}$ and $\mathrm{t}_{\mathrm{N}} \in \mathrm{R}$ such that:

$$
\Phi\left(\mathrm{t}_{\mathrm{N}}+\mathrm{s}_{\mathrm{n}_{\mathrm{N}}}\right)-\Phi\left(\mathrm{t}_{\mathrm{N}}+\mathrm{s}_{\mathrm{m}_{\mathrm{N}}}\right) \notin \mathrm{u} \text {. }
$$


By Bochner's criteria we can extract two subsequences $\left(s^{\prime}{ }_{n_{N}}\right) \subset\left(s_{n_{N}}\right)$ and $\left(\mathrm{s}^{\prime} \mathrm{m}_{\mathrm{N}}\right) \subset\left(\mathrm{s}_{\mathrm{m}_{\mathrm{N}}}\right)$ such that

$$
\begin{aligned}
& \lim _{N \rightarrow \infty} \Phi\left(t+s^{\prime} n_{N}\right)=g_{1}(t) \text { uniformly in } t \varepsilon R, \\
& \lim _{N \rightarrow \infty}\left(t+s^{\prime} m_{N}\right)=g_{2}(t) \text { uniformly in } t \varepsilon R .
\end{aligned}
$$

Let $\mathrm{V}$ be a symmetric neighbourhood with $\mathrm{V}+\mathrm{V}+\mathrm{V} \subset \mathrm{U}$. Then there exists $\mathrm{N}_{\mathrm{O}}=\mathrm{N}_{\mathrm{O}_{\mathrm{V}}}$ such that if $\mathrm{N}>\mathrm{N}_{0}$,

$$
\begin{aligned}
& \Phi\left(t_{N}+s^{\prime}{ }_{n_{N}}\right)-g_{1}\left(t_{N}\right) \varepsilon v, \\
& \Phi\left(t_{N}+s^{\prime}{ }_{m_{N}}\right)-g_{2}\left(t_{N}\right) \varepsilon v .
\end{aligned}
$$

We conclude $g_{1}\left(t_{N}\right)-g_{2}\left(t_{N}\right) \notin V$. If not, we should get

$$
\begin{aligned}
\Phi\left(t_{N}+s^{\prime} n_{N}\right)-\Phi\left(t_{N}+s^{\prime} m_{N}\right)= & \Phi\left(t_{N}+s^{\prime} n_{N}\right)-g_{1}\left(t_{N}\right) \\
& +g_{1}\left(t_{N}\right)-g_{2}\left(t_{N}\right) \\
& +g_{2}\left(t_{N}\right)-\Phi\left(t_{N}+s^{\prime} m_{N}\right)
\end{aligned}
$$

and therefore $\Phi\left(t_{N}+s^{\prime} n_{N}\right)-\Phi\left(t_{N}+s^{\prime} m_{N}\right) \varepsilon U$; this contradicts (1).

We have found $\mathrm{V}$ with the property that if $\mathrm{N}$ is large enough, there exists $t_{N} \varepsilon R$ such that

$$
g_{1}\left(t_{N}\right)-g_{2}\left(t_{N}\right) \notin V
$$

But this is impossible; because if we take a subsequence $\left(\xi_{k}\right)_{k=1}^{\infty} \subset\left(n_{k}\right)_{k=1}^{\infty}$ and $\xi_{k} \rightarrow t_{N}$, then we have

$$
\lim _{\mathrm{N} \rightarrow \infty} \Phi\left(\xi_{\mathrm{k}}+\mathrm{s}^{\prime} \mathrm{n}_{\mathrm{N}}\right)=\lim _{\mathrm{N} \rightarrow \infty} \Phi\left(\xi_{\mathrm{k}}+\mathrm{s}^{\prime}{ }_{\mathrm{m}_{\mathrm{N}}}\right)
$$

for every $k$, and therefore $g_{1}\left(\xi_{k}\right)=g_{2}\left(\xi_{k}\right)$ for every $k$; by continuity of $g_{1}$ and $g_{2}$, $g_{1}\left(t_{N}\right)=g_{2}\left(t_{N}\right)$, thus $g_{1}\left(t_{N}\right)-g_{2}\left(t_{N}\right)$ belongs to every neighbourhood of 0 . The lemma is proved.

Let us now continue proving theorem 8. Consider arbitrary real sequences $\left(h_{n}\right)_{n=1}^{\infty}$ and $\left(n_{r}\right)_{r=1}^{\infty}$ the rational numbers.

By relative compacity of $\{f(t), t \varepsilon R\}$, we can extract a subsequence $\left(h_{n}\right)_{n=1}^{\infty}$ (we do not change notation) such that for each $r$,

$$
\lim _{n \rightarrow \infty} f\left(n_{r}+h_{n}\right)=x_{r} \text { exists in } E \text {. }
$$

Now $\left(f\left(n_{r}+h_{n}\right)\right)_{n=1}^{\infty}$ is uniformly convergent in $r$. Suppose it is not; then we find a neighbourhood $U$ and three subsequences $\left(\xi_{r}\right)_{r=1}^{\infty} \subset\left(n_{r}\right)_{r=1}^{\infty},\left(h^{\prime}{ }_{r}\right)_{r=1}^{\infty}:\left(h_{r}\right)_{r=1}^{\infty}$, $\left(h^{\prime \prime}{ }_{r}\right)_{r=1}^{\infty} \subset\left(h_{r}\right)_{r=1}^{\infty}$ and

$$
f\left(\xi_{r}+h^{\prime}{ }_{r}\right)-f\left(\xi_{r}+h^{\prime \prime}\right) \notin U \text {. }
$$

By relative compacity of $\{f(t) ; t \varepsilon R\}$ we may say

$$
\begin{aligned}
& \lim _{r \rightarrow \infty} f\left(\xi_{r}+h^{\prime}{ }_{r}\right)=b^{\prime} \varepsilon E, \\
& \lim _{r \rightarrow \infty} f\left(\xi_{r}+h^{\prime \prime}{ }_{r}\right)=b^{\prime \prime} \varepsilon E,
\end{aligned}
$$

and using (4.5), we get

$$
\mathrm{b}^{\prime}-\mathrm{b}^{\prime \prime} \notin \mathrm{U} \text {. }
$$

By the Hahn-Banach theorem, there exists $x^{*} \varepsilon E^{*}$ such that

$$
x^{*}\left(b^{\prime}\right) \neq x *\left(b^{\prime \prime}\right) \text {. }
$$


But $f(t)$ is w.a.p. hence $\left(x^{*} f\right)(t)$ is a.p. and consequently it is uniformly continuous over R.

Consider the sequence of functions $\left(\varphi_{n}\right)_{n=1}^{\infty}$ defined by:

$$
\varphi_{n}(t)=\left(x^{*} f\right)\left(t+h_{n}\right), n=1,2, \ldots
$$

The equality $\varphi_{n}(t+\tau)-\varphi_{n}(t)=x^{*} f\left(t+\tau+h_{n}\right)-x * f\left(t+h_{n}\right)$ shows almost-periodicity of each $\varphi_{n}$. Also $\left(\varphi_{n}\right)_{n=1}$ is equi-uniformly continuous over $R$ because (x*f) is uniformly continuous over $R$, as it is easy to see. Using (4.4), we can say

$$
\lim _{n \rightarrow \infty} x * f\left(n_{r}+h_{n}\right)=x *\left(x_{r}\right)
$$

for every $r$. Therefore, by lemma $1,\left(x^{*} \mathrm{f}\left(\mathrm{t}+\mathrm{h}_{\mathrm{n}}\right)\right)_{\mathrm{n}=1}^{\infty}$ is uniformly convergent in $t$. Consider now the sequences $\left(\xi_{r}+h^{\prime}{ }_{r}{ }^{\infty} r=1\right.$ and $\left(\xi_{r}+h^{\prime \prime}{ }_{r}\right)_{r=1}^{\infty}$. By Bochner's criteria, we extract two subseqeunces ( we use the same notations) such that $\left(x * f\left(t+\xi_{r}+h^{\prime}{ }_{r}\right)\right)_{r=1}^{\infty}$ and $\left(\mathrm{x} * \mathrm{f}\left(\mathrm{t}+\mathrm{r}+\mathrm{h}_{\mathrm{r}}\right)\right)_{\mathrm{r}=1}^{\infty}$ are uniformly convergent in $\mathrm{t} \in \mathrm{R}$.

Let us now prove

$$
\lim _{\mathbf{r} \rightarrow \infty} x^{*} f\left(t+\xi_{r}+h^{\prime}{ }_{r}\right)=\lim _{r \rightarrow \infty} x * f\left(t+\xi{ }_{r}+h^{\prime \prime}\right)
$$

Consider the inequality:

$$
\begin{aligned}
\mid x^{* f}\left(t+\xi_{r}+h^{\prime}{ }_{r}\right) & -x^{* f}\left(t+\xi_{r}+h^{\prime \prime}\right) \mid \\
& \leq\left|x * f\left(t+\xi_{r}+h^{\prime}{ }_{r}\right)-x * f\left(t+\xi_{r}+h_{r}\right)\right| \\
& +\left|x^{*} f\left(t+\xi_{r}+h_{r}\right)-x * f\left(t+\xi_{r}+h^{\prime \prime}{ }_{r}\right)\right|
\end{aligned}
$$

$\mathbf{r}=1,2, \ldots$

Let $\varepsilon>0$ be given; as $\left(x * f\left(t+h_{r}\right)\right)_{r=1}^{\infty}$ is uniformly convergent in $t$, we choose $n_{\varepsilon}$ such that for $r, s>n_{\varepsilon}$, we have $\left|x^{* f}\left(t+h_{s}\right)-x^{*} f\left(t+h_{r}\right)\right|<\frac{\varepsilon}{2}$, for $t \varepsilon k$; then for $r, s>n_{\varepsilon}$, we get

$$
\left|x * f\left(t+\xi_{r}+h_{s}\right)-x * f\left(t+\xi_{r}+h_{r}\right)\right|<\frac{\varepsilon}{2} \text {. }
$$

Consequently, for $r>n_{\varepsilon}$, we get:

$$
\begin{aligned}
& \left|x * f\left(t+\xi_{r}+h^{\prime}\right)-x * f\left(t+\xi_{r}+h_{r}\right)\right|<\frac{\varepsilon}{2}, \\
& \left|x * f\left(t+\xi_{r}+h^{\prime \prime}\right)-x * f\left(t+\xi_{r}+h_{r}\right)\right|<\frac{\varepsilon}{2}
\end{aligned}
$$

and the inequality $(4.10)$ gives:

$$
\left|x^{*} f\left(t+\xi_{r}+h^{\prime}{ }_{r}\right)-x * f\left(t+\xi_{r}+h^{\prime \prime}{ }_{r}\right)\right|<\varepsilon
$$

for $t \in R$. (4.9) is then proved.

Now take $t=0$; then using (4.6) we get:

$$
x^{*}\left(b^{\prime}\right)=\lim _{r \rightarrow \infty} x^{* f}\left(\xi_{r}+h^{\prime}\right)=\lim _{r \rightarrow \infty} x^{* f}\left(\xi_{r}+h^{\prime \prime}{ }_{r}\right)=x^{*}\left(b^{\prime \prime}\right)
$$

which contradicts $(4.8)$ and uniform convergence in $r$ for $\left(f\left(n_{r}+h_{n}\right)\right)_{n=1}^{\infty}$.

If $i, j>N$, we have

$$
f\left(n_{r}+h_{i}\right)-f\left(n_{r}+h_{u}\right) \varepsilon U \text {, for every } r \text {. }
$$

Therefore if $t \in R$, we take a subsequence of $\left(n_{r}\right)_{r=1}^{\infty}$ which converges to $t$ and using continuity of $f$ and the relation (4.11), we obtain, for $i, j>N$,

$f$ is then a.p..

$$
f\left(t+h_{i}\right)-f\left(t+h_{j}\right) \varepsilon U .
$$

THEOREM 9. Let $\mathrm{E}$ be a Fréchet space. If $\mathrm{f}: \mathrm{R} \rightarrow \mathrm{E}$ is a.p. and $\{\mathrm{F}(\mathrm{t}) ; \mathrm{t} \varepsilon \mathrm{R}\}$ where $F(t)=\int_{0}^{t} f(\sigma) d \sigma$ is relatively compact in $E$, then $F$ is a.p.. 
PROOF. Immediate from theorems 7 and 8 .

THEOREM 10. Let $E$ be a complete 1.c.s.. If $f$ is a.p. and its derivative $f^{\prime}$ uniformly continuous over the real line, then $f^{\prime}$ is also a.p..

PROOF. Consider the sequence of a.p. functions $\left\{n\left(f\left(t+\frac{1}{n}\right)-f(t)\right)\right\}_{n=1}^{\infty}$; it suffices to prove it converges uniformly over the real line to $f^{\prime}(t)$.

Let $U=U\left(\varepsilon ; p_{i}, 1 \leq i \leq n\right)$; by uniform continuity of $\mathrm{f}^{\prime}$, we can choose $\delta=\delta_{U}>0$ such that $f^{\prime}\left(t_{1}\right)-f^{\prime}\left(t_{2}\right) \varepsilon U$ for every $t_{1}, t_{2}$ with $\left|t_{1}-t_{2}\right|<0$. We write

$$
f^{\prime}(t)-n\left(f\left(t+\frac{1}{n}\right)-f(t)\right)=n \int_{0}^{\frac{1}{n}}\left[f^{\prime}(t)-f^{\prime}(t+\sigma)\right] d \sigma .
$$

Therefore, if we take $\mathrm{N}=\mathrm{N}_{\mathrm{U}}>\frac{1}{\delta}$, then for $\mathrm{n}, \mathrm{N}$, we have:

$$
p_{i}\left[f^{\prime}(t)-n\left(f\left(t+\frac{1}{n}\right)-f(t)\right)\right] \leq n \int_{0}^{\frac{1}{n}} p_{i}\left[f^{\prime}(t)-f^{\prime}(t+\sigma)\right] d \sigma<\varepsilon
$$

for every semi-norm $p_{i}$ and every $t \varepsilon R$. ithe theorem is proved.

THEOREM 11. Let $\mathrm{E}$ be a Fréchet space; then the set of all a.p. $\mathrm{f}: \mathrm{R} \rightarrow \mathrm{E}$ is a Banach space under the supremum norm.

PROOF. Obvious; use theorems 1,2 and 6 .

5. APPLICATIONS TO ABSTRACT DIFFERENTIAL EQUATIONS

A. A.P. SOLUTIONS OF $\left(\frac{d}{d x}-A\right) x=0$

Consider in a complete 1.c.s. E the differential equation

$$
\frac{d x}{d t}=A x(t),-\infty<t<\infty,
$$

where $A$ is a continuous linear operator such that $\left\{A^{k} ; k=1,2, \ldots\right\}$ is equi-continuous. A solution of $(5.1)$ is a continuous $1 y$ differentiable function which satisfies (5.1). It is easy to construct (as in [13] p. 244-246) a solution of the form:

$$
e^{t A} x(0)=\sum_{k=0}^{\infty} \frac{t^{k}}{k !} A^{k} x(0)
$$

We can say more:

PROPOSITION 1. The function $e^{t A} x_{0}: R \rightarrow E$ is the unique solution of the Cauchy problem:

$$
\begin{aligned}
& \frac{d x}{d t}=A x(t) ;-\infty<t<\infty, \\
& x(0)=x_{0} .
\end{aligned}
$$

PROOF. Suppose there exists another solution $y(t)$ with $y(0)=x_{0}$; consider the function $v(\tau)=e^{(t-\tau) A} y(\tau)$, with fixed $t$ and show it is constant over the real line; therefore $v(\tau)=v(0)$ for every $\tau \varepsilon R$, which means $v(t)=v(0)$, or $y(t)=e^{t A} x_{0}$, proving uniqueness of the solution (see[9] for a complete proof).

Now, define a perfect Fréchet space $E$ as a Fréchet space with the following property: every function $f: R \rightarrow E$ with (i) $\{f(t) ; t \varepsilon R\}$ is bounded in $E$; (ii) $\mathrm{f}^{\prime}(\mathrm{t})$ is a.p. in $\mathrm{E}$; is necessarilya.p. in $\mathrm{E}$.

We state and prove the two following theorems inspired from a result of PEKOV (see [15] theorem 1.1) but they are not direct generalisations. In fact they are new results.

THEOREM 1. Let $E$ be a perfect Fréchet space; assume (i) A is a compact linear operator; (ii) $\left\{A^{k} ; k=1,2, \ldots\right\}$ is equi-continuous; (iii) for every semi-norm $p$, there exists a semi-norm $q$ such that $p\left[e^{t A} x\right] \leq q(x)$ for every $x \varepsilon E$ and every $t \varepsilon R$. 
Then every solution $x(t)$ of $(5.1)$ is a.p. in E.

PROOF. Because $x(t)=e^{t A} x(0)$, then $x(t)$ is bounded in $E$ by (iii). E being a perfect Fréchet space, it suffices to prove $x^{\prime}(t)$ is a.p..

$\{A x(t) ; t \varepsilon R\}$ is also relatively compact in $E$ fur $A$ is a compact operator; consequently $\{x(t) ; t \varepsilon R\}$ is also relatively compact. Let $\left(s^{\prime}{ }_{n}\right)_{n=1}^{\infty}$ be an arbitrary real sequence; we then can extract a subsequence $\left(s_{n}\right)_{n=1}^{\infty}$ such that $\left(x^{\prime}\left(s_{n}\right)\right)_{n=1}^{\infty}$ is a Cauchy sequence in $E$. But we have:

$$
\begin{aligned}
x^{\prime}\left(t+s_{n}\right) & =A x\left(t+s_{n}\right)=A e^{\left(t+s_{n}\right) A} x(0)=A e^{t A} e^{s} A_{x}(0) \\
& =A e^{t A} x\left(s_{n}\right)=e^{t A} A x\left(s_{n}\right)=e^{t A} x^{\prime}\left(s_{n}\right)
\end{aligned}
$$

for every $n=1,2, \ldots$, and every $t \varepsilon R$. If $p$ is a given semi-norm, there exists a semi-norm q such that

$$
\begin{aligned}
\mathrm{p}\left[\mathrm{x}^{\prime}\left(\mathrm{t}+\mathrm{s}_{\mathrm{n}}\right)-\mathrm{x}^{\prime}\left(\mathrm{t}+\mathrm{s}_{\mathrm{m}}\right)\right] & =\mathrm{p}\left[\mathrm{e}^{\mathrm{tA}}\left(\mathrm{x}^{\prime}\left(\mathrm{s}_{\mathrm{n}}\right)-\mathrm{x}^{\prime}\left(\mathrm{s}_{\mathrm{m}}\right)\right)\right] \\
& \leq \mathrm{q}\left[\mathrm{x}^{\prime}\left(\mathrm{s}_{\mathrm{n}}\right)-\mathrm{x}^{\prime}\left(\mathrm{s}_{\mathrm{m}}\right)\right]
\end{aligned}
$$

for every $t \in R$ and every $n, m \varepsilon N$. Therefore $\left(x^{\prime}\left(t+s_{n}\right)\right)_{n=1}^{\infty}$ is uniformly Cauchy in $t$; we then conclude almost-periodicity of $x^{\prime}(t)$ by Bochner's criteria.

THEOREY 2. Let $E$ be a Fréchet space; assume conditions (1) - (iii) in theorem 1 are satisfied and moreover the range $R(A)$ of $A$ is dense in $E$. Then every solution $x(t)$ of $(5.1)$ is a.p. in E.

We remark the first part of the proof of theorem 1 tells us if $x(t)$ is a solution of (5.1) with $x(0) \varepsilon D(A)=E$, then $x^{\prime}(t)$ is a.p.. Before proving Theorem 2 let us state and prove:

LEMMA 1. Every solution of (j.1) with initial data in $R(A)$ is a.p..

PROOF. Let a $\varepsilon K(A)$ and consider the solution $y(t)$ with $y(0)=a$; there exists $x_{0} \in D(A)=E$ such that $A x_{0}=a$. We have $y(t)=e^{t A} a=e^{t A} A x_{0}=a e^{t A} x_{0}=A x(t)=$ $x^{\prime}(t)$ where $x(t)=e^{t A} x_{0}$; therefore $x^{\prime}(t)$ (and consequently $y(t)$ ) is a.p.. The lemma is proved.

PROOF OF THEOREM 2. Consider a solution $x(t)$ of $(5.1)$ with $x(0) \varepsilon E$; as $R(A)$ is dense in $E$, there exists a sequence $\left(a_{n}\right)_{n=1}^{\infty}$ in $R(A)$ such that $a_{n} \rightarrow x(0)$. Consider a sequence of solutions $\left(y_{n}(t)\right)_{n=1}^{\infty}$ with $y_{n}(0)=a_{n}, n=1,2, \ldots$ To prove almostperiodicity of $x(t)$ it suffices to prove $y_{n}(t) \rightarrow x(t)$ uniformly in $t \varepsilon$ for every $y_{n}(t)$ is a.p. by 1emma 1 . We have $x(t)=e^{t A} x(0), y_{n}(t)=e^{t A} a_{n}, n=1,2, \ldots$ Now given a semi-norm $p$ there exists, by assumption (iii), a semi-norm $q$ such that $p\left(y_{n}(t)-x(t)\right) \leq q\left(a_{n}-x(0)\right)$, for every $t \varepsilon R$ and every $n \varepsilon N$. The conclusion is immediate.

B. A.P. SOLUTIONS OF $\left(\frac{d}{d x}-A\right) x=f$

We now consider the non-homogeneous differential equation

$$
\frac{d x}{d t}=A x(t)+f(t),-\infty<t<\infty
$$

where $A$ is a closed linear operator with domain $D(A)$ dense in a Fréchet space E; the function $f(t)$ is a.p. in $E$. Let us recall some useful definitions (see 13 ). A family of continuous linear operators $T(t), t \varepsilon R$, is an equi-continuous $\mathrm{C}_{0}$-group: 
(i) $\mathrm{T}\left(\mathrm{t}_{1}+t_{2}\right) \mathrm{x}=\mathrm{T}\left(\mathrm{t}_{1}\right) \mathrm{T}\left(\mathrm{t}_{2}\right) \mathrm{x}, \mathrm{T}(0) \mathrm{x}=\mathrm{x}$, for every $\mathrm{x} \varepsilon \mathrm{E}$ and every $t_{1}, t_{2} \varepsilon \mathrm{R}$;

(ii) for every semi-norm $p$, there exists a semi-norm $q$ such that $p[T(t) x] \leq q(x)$ for every $x \in E$ and every $t \in R$.

(iii) $\lim _{t \rightarrow t_{0}} T\left(t_{0}\right) x$, for every $x \in E$ and every $t_{0} \varepsilon R$.

Now consider an equi-continuous $C$-group $T(t)$. A is called the infinitesimal generator of $T(t)$ if $A x=\lim _{n \rightarrow 0} \frac{T(n) x-0 x}{n}$, i.e., A is the linear operator with domain $D(A)=\left\{x \in E ; \lim _{n \rightarrow 0} \frac{T(\eta) x-x}{\eta}\right.$ exists in $\left.E\right\}$ and for every $x \in D(A), A x=\lim _{\eta \rightarrow 0} \frac{T(\eta) x-x}{\eta}$. It can be proved $\frac{d}{d t} T(t) x=A T(t) x=T(t) A x$ for every $x \in D(A)$ (see[13] for the case of a semi-group).

We are going to prove the following theorem 3 which is a generalization of theorem 3.2 [15] due to ZAIDMAN.

THEOREM 3. Let $E$ be a Fréchet space. Suppose $x(t)$ is a solution of equation (5.3) with relatively compact trajectory; $A$ is the infinitesimal generator of equicontinuous $C_{0}$-group $T(t)$ such that $T(t) x: R \rightarrow E$ is a.p. for every $x \in E ; f(t)$ is a.p.. Then $x(t)$ is also a.p..

Before we prove theorem 3, let us mention some useful lemmas (see [9] for proofs):

LEMMA 2. Let $E$ be a complete 1.c.s.. If $f(\sigma)$ is continuous, then $T(t-\sigma) f(\sigma)$ : $\mathrm{R} \rightarrow \mathrm{E}$ is also continuous for every $\mathrm{t} \varepsilon \mathrm{R}$.

LEMMA 3. In a complete 1.c.s. E, every solution of (5.3) admits the integral representation:

$$
x(t)=T(t) x(0)+\int_{0}^{t} T(t-\sigma) f(\sigma) d .
$$

LEMMA 4. Let $E$ be a Fréchet space. If $\{T(t) x ; t \varepsilon R\}$ is relatively compact in $E$ for every $x \in E$ and $\{f(t) ; t \varepsilon R\}$ is also relatively compact in $E$, then $\{T(t) f(t)$; $t \varepsilon R\}$ is relatively compact in $E$.

PROOF. Let $\left(t^{\prime \prime}\right)_{n=1}^{\infty}$ be an arbitrary real sequence; by our assumption on $f(t)$, we can extract a subsequence $\left(t^{\prime}{ }_{n}\right)_{n=1}^{\infty} \subset\left(t^{\prime \prime}{ }_{n}{ }_{n=1}\right.$ such that $\lim _{n \rightarrow \infty} f\left(t^{\prime}{ }_{n}\right)$ exists in $E$; let $\mathrm{x}$ be this limit.

Take another subsequence $\left(t_{n}\right)_{n=1}^{\infty} \subset\left(t^{\prime}{ }_{n}\right)_{n=1}^{\infty}$ such that $\left(T\left(t_{n}\right) x\right)_{n=1}^{\infty}$ is a Cauchy sequence in $E$. Write:

$$
\begin{aligned}
T\left(t_{n}\right) f\left(t_{n}\right)-T\left(t_{m}\right) f\left(t_{m}\right) & =\left[T\left(t_{n}\right)-T\left(t_{m}\right)\right]\left[f\left(t_{n}-x\right]\right. \\
& +\left[\left(T\left(t_{n}\right)-T\left(t_{m}\right)\right) x\right] \\
& +T\left(t_{m}\right)\left[f\left(t_{n}\right)-f\left(t_{m}\right)\right] .
\end{aligned}
$$

Let $\mathrm{p}$ be any semi-norm; then we have

$$
\begin{aligned}
p\left[T\left(t_{n}\right) f\left(t_{n}\right)-T\left(t_{m}\right) f\left(t_{m}\right)\right] & <p\left[\left[T\left(t_{n}\right)-T\left(t_{m}\right)\right]\left[f\left(t_{n}\right)-x\right]\right] \\
& \left.+p\left[T\left(t_{n}\right)-T\left(t_{m}\right)\right) x\right] \\
& +p\left[T\left(t_{m}\right)\left[f\left(t_{n}\right)-f\left(t_{m}\right)\right]\right] .
\end{aligned}
$$

Using equi-continuity of $\mathrm{T}(\mathrm{t})$, we can take a semi-norm $q$ such that

$$
p\left[T\left(t_{n}\right)\left[f\left(t_{n}\right)-f\left(t_{m}\right)\right]\right] \leq q\left[f\left(t_{n}\right)-f\left(t_{m}\right)\right]
$$

and

$$
p\left[\left[T\left(t_{n}\right)-T\left(t_{m}\right)\right]\left[f\left(t_{n}\right)-x\right]\right] \leq 2 q\left[f\left(t_{n}\right)-x\right] .
$$

Now we choose $\mathrm{n}$ and $\mathrm{m}$ sufficiently large such that

$$
q\left[f\left(t_{n}\right)-f\left(t_{m}\right)\right]<\frac{\varepsilon}{3}, q\left[f\left(t_{n}\right)-x\right]<\frac{\varepsilon}{6}, p\left[\left(T\left(t_{n}\right)-T\left(t_{m}\right)\right) x\right]<\frac{\varepsilon}{3}
$$


then we obtain:

$$
p\left[T\left(t_{n}\right) f\left(t_{n}\right)-T\left(t_{m}\right) f\left(t_{m}\right)\right]<\varepsilon
$$

which shows $\left(T\left(t_{n}\right) f\left(t_{n}\right)\right)_{n=1}^{\infty}$ is a Cauchy sequence. The lemma is proved.

LEMMA 5. Let $E$ be a Frechet space and consider the equi-continuous $\mathrm{C}_{0}$-group $T(t)$ such that $T(t) x: R \rightarrow E$ is a.p. for every $x \in E$. Suppose also $f(t)$ is a.p.. Then $T(t) f(t): R \rightarrow E$ is a.p..

PROOF. Consider $U=U\left(\varepsilon ; p_{i}, 1 \leq i \leq n\right)$ a given neighbourhood; because of equicomtinuity of $T(t)$, there corresponds to each semi-norm $p_{i}$, a semi-norm $q_{1}$ such that: (i) $\mathrm{p}_{i}(\mathrm{~T}(\mathrm{t}) \mathrm{x}) \leq \mathrm{q}_{\mathrm{i}}(\mathrm{x}), \mathrm{x} \in \mathrm{E}, \mathrm{t} \varepsilon \mathrm{R}$.

Consider also the symmetric neighbourhood

$$
\mathrm{V}=\mathrm{V}\left(\frac{\varepsilon}{4} ; \mathrm{p}_{\mathrm{i}}, \mathrm{q}_{\mathrm{i}}, 1 \leq \mathrm{i} \leq \mathrm{n}\right) ; \mathrm{V}+\mathrm{v}+\mathrm{v}+\mathrm{V} \subset \mathrm{U} \text {. }
$$

As $\{f(t) ; t \varepsilon R\}$ is totally bounded, there exists $t_{1}, \ldots, t_{\nu}$ such that for every $t \varepsilon R$ we have $f(t) \quad \varepsilon \bigcup_{k=1}^{U}\left(f\left(t_{k}\right)+v\right)$. Consider now the following a.p. functions: $f(t)$, $\mathrm{T}(\mathrm{t}) \mathrm{f}\left(\mathrm{t}_{\mathrm{k}}\right), \mathrm{k}=1, \ldots, v$. Then they have the same $\mathrm{V}$-translation numbers; therefore we can say there exists $\ell=\ell(V)>0$ such that any interval $[a, a+\ell]$ contains $\tau$ with

$$
\begin{aligned}
& f(t+\tau)-f(t) \varepsilon V, t \varepsilon R \\
& T(t+\tau) f\left(t_{k}\right)-T(t) f\left(t_{k}\right) \varepsilon V, k=1, \ldots, t \varepsilon R .
\end{aligned}
$$

Take $t \varepsilon R$ arbitrary; then there exists $k(1 \leq k \leq v)$ such that

$$
f(t) \varepsilon f\left(t_{k}\right)+v \text {. }
$$

Write:

$$
\begin{aligned}
& T(t+\tau) f(t+\tau)-T(t) f(t)=\{T(t+\tau)[f(t+\tau)-f(t)]\} \\
+ & \left\{?(t+\tau)\left[f(t)-f\left(t_{k}\right)\right]\right\}+\left\{T(t+\tau) f\left(t_{k}\right)-T(t) f\left(t_{k}\right)\right\} \\
+ & \left\{T(t)\left[f\left(t_{k}\right)-f(t)\right]\right\} .
\end{aligned}
$$

For every semi-norm $p_{i}$; there exists a semi-norm $q_{i}$ such that:

$p_{i}[T(t+\tau) f(t+\tau)-T(t) f(t)] \leq q_{i}[f(t+\tau)-f(t)]$

$+q_{i}\left[f(t)-f\left(t_{k}\right)\right]+p_{i}\left[T(t+\tau) f\left(t_{k}\right)-T(t) f\left(t_{k}\right)\right]$

$+q_{i}\left[f\left(t_{k}\right)-f(t)\right]<\frac{\varepsilon}{4}+\frac{\varepsilon}{4}+\frac{\varepsilon}{4}+\frac{\varepsilon}{4}=\varepsilon$, (using (5.3)- (5.5).

Therefore $T(t+\tau) f(t+\tau)-T(t) f(t) \varepsilon U$ for every $t \varepsilon R$, which is almost-periodicity for $T(t) f(t)$.

PROOF OF THEOREM 3. By lemma 3 we have : $x(t)=T(t) x(0)+\int_{0}^{t} T(t-\sigma) f(\sigma) d \sigma$. But $T(t) x(0)$ is a.p.. It remains to prove the function $v(t)=\int_{0}^{+t} T(t-\sigma) f(\sigma) d \sigma$
is also a.p..

As $\{x(t) ; t \varepsilon R\}$ and $\{T(t) x(0) ; t \varepsilon R\}$ are relatively compact, then $\{v(t) ; t \varepsilon R\}$ also is relatively compact. Let us write $v(t)=\int_{0}^{t} T(t) T(-\sigma) f(\sigma) d \sigma$

$=T(t) \int_{0}^{t} T(-\sigma) f(\sigma) d \sigma$. Then $T(-t) v(t)=\int_{0}^{t} T(-\sigma) f(\sigma) d \sigma$.

By theorem 3 of chapter $1, T(-t) x$ is a.p. for every $x \varepsilon E$, therefore $\{T(-t) x$; $t \varepsilon R\}$ is relatively compact for every $x \in E$. By lemma $4,\{T(-t) v(t) ; t \varepsilon R\}$ and consequently $\left\{\int_{0}^{\mathrm{t}} \mathrm{T}(-\sigma) \mathrm{f}(\sigma) \mathrm{d} \sigma ; \mathrm{t} \varepsilon \mathrm{R}\right\}$ is relatively compact. By lemma $5, \mathrm{~T}(-\mathrm{t}) \mathrm{f}(\mathrm{t})$ is a.p., therefore $\int_{0}^{t} \mathrm{~T}(-\sigma) \mathrm{f}(\sigma) \mathrm{d} \sigma$ is a.p.. We apply again lemma 5 to conclude almostperiodicity of $\int_{0}^{t} \mathrm{~T}(t-\sigma) \mathrm{f}(\sigma) \mathrm{d} \sigma$. Theorem 3 is proved. 
THEOREM 4. Let $E$ be a Fréchet space. Solutions of the equation $x^{\prime}(t)=A x(t)$, $-\infty<t<\infty$, with relatively compact trajectory are precisely almost-periodic ones, if $A$ is the infinitesimal generator of equi-continuous $C_{0}$-group $T(t)$.

PROOF. Let $x(t)$ be a solution of the given equation. It suffices to prove that if $x(t)$ has a relatively compact trajectory, then $x(t)$ is a.p.. Take an arbitrary real sequence $\left(s^{\prime}{ }_{n}\right)_{n=1}^{\infty}$; we can extract a subsequence $\left(s_{n}\right)_{n=1}^{\infty} \subset\left(s_{n}^{\prime}\right)_{n=1}^{\infty}$ such that $\left(x\left(s_{n}\right)\right)_{n=1}^{\infty}$ is a Cauchy sequence in $E$; but we have

$$
x\left(t+s_{n}\right)=T\left(t+s_{n}\right) x(0)=T(t) T\left(s_{n}\right) x(0)=T(t) x\left(s_{n}\right), n=1,2, \ldots
$$

Therefore

$$
x\left(t+s_{n}\right)-x\left(t+s_{m}\right)=T(t)\left[x\left(s_{n}\right)-x\left(s_{m}\right)\right], n, m \varepsilon N .
$$

Let $p$ be a given semi-norm; by equi-continuity of $T(t)$, there exists a semi-norm $q$ such that:

$$
\mathrm{p}\left[\mathrm{x}\left(\mathrm{t}+\mathrm{s}_{\mathrm{n}}\right)-\mathrm{x}\left(\mathrm{t}+\mathrm{s}_{\mathrm{m}}\right)\right] \leq \mathrm{q}\left[\mathrm{x}\left(\mathrm{s}_{\mathrm{n}}\right)-\mathrm{x}\left(\mathrm{s}_{\mathrm{m}}\right)\right], t \varepsilon R .
$$

Which shows $\left(x\left(t+s_{n}\right)\right)_{n=1}^{\infty}$ is a Cauchy sequence, uniform in $t \varepsilon R$. We conclude using Bochner's criteria.

ACKNOWLEUGEMENT. We would like to thank Professor S. ZAIDMAN for the useful discussions we had during this work and Professor J.A. GULDSTEIN for suggesting theorem 2 in section 5 and encouraging me to publish these results.

\section{REFERENCES}

1. BOCHNER, S., Continuous Mappings of Almost-Automorphic and Almost-Periodic Functions, Proc. Nat. Acad. Sc. 52 (1964).

2. BOCHNER, S. and von NEUMANN, J., Almost-Periodic Functions in Groups, Trans. Amer. Math. Soc. 37 (1935), 21-50.

3. AMERIO, L., Funzioni Debolmente Quasi-Periodiche, Rend. Sem. Mat. Padova 30 (1960).

4. AMERIO, L. and PROUSE, G., Almost-Periodic Functions and Functional Equations. Van Nostrand 1971.

5. BESICUVI'CH, A.S., Almost-Periodic Functions, Dover.

6. CORDUNEANU, C., Almost-Periodic Functions, Interscience, 1968.

7. FINK, A.M., Almost-Periodic Differential Equations, Springer Verlag, 1974.

8. ROBERTSON, A.P. and ROBERTSON, W., Topological Vector Spaces, Cambridge University Press, 1973.

9. NGUEREKATA, G.M., Remarques sur les équations différentielles abstraites, Thèse de $\mathrm{Ph}$. D. Université de Montréal, Juin 1980.

10. HILLE, E. and PHILIPS, R.S., Functional Analysis and Semi-Groups, A.M.S. Colloquium Publications, 31 (195\%).

11. MIYADERA, I., Semi-Groups of Operators in Fréchet Space and Applications to Partial Differential Equations, Math. Inst., Tokyo Metro Univ. (1958) 162-183.

12. NEUMANN von, J., Almost-Periodic Functions in a Group, Trans. Amer. Math. Soc. 36 $(1934), 445-492$.

13. YOS IDA, K., Functional Analysis, Springer Verlag, 1968.

14. ZAIDMAN, S., Some Remarks on Almost-Periodicity, Atti della Academia delle scienze di Torino 106 (1971-72).

15. ZAIDMAN, S., Solutions Presque-Périodiques des Équations Différentielles Abstraites, L'enseignement Math. $24(19 \%), 87-110$. 


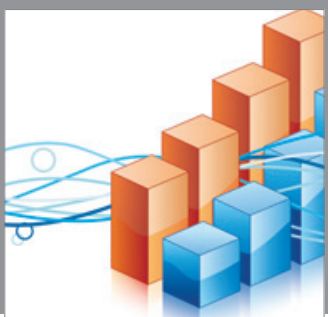

Advances in

Operations Research

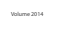

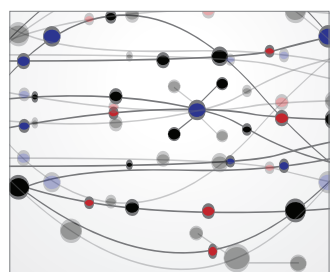

\section{The Scientific} World Journal
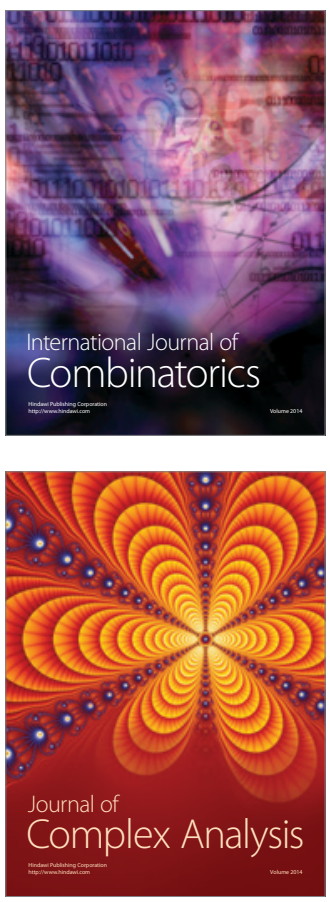

International Journal of

Mathematics and

Mathematical

Sciences
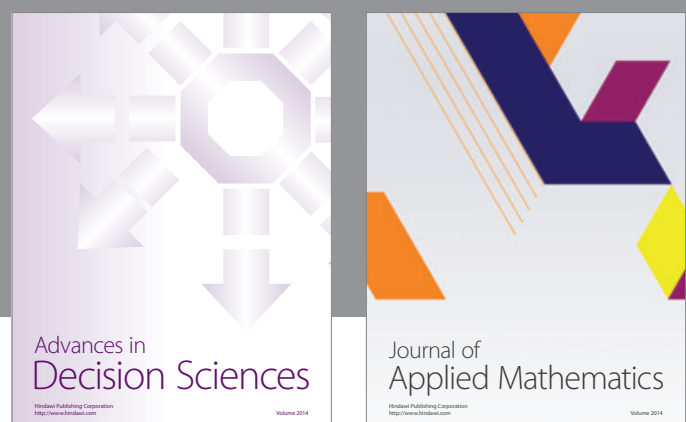

Journal of

Applied Mathematics
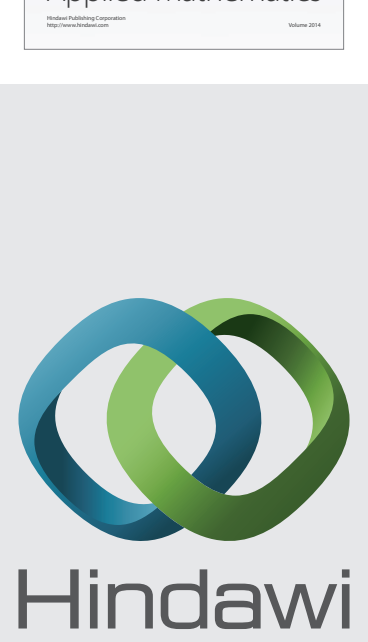

Submit your manuscripts at http://www.hindawi.com
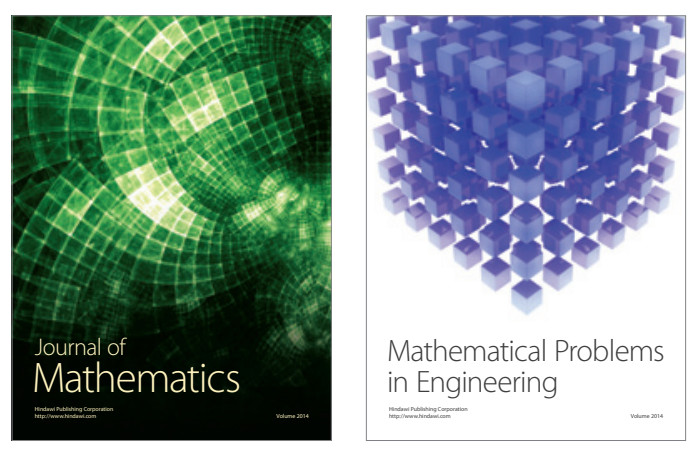

Mathematical Problems in Engineering
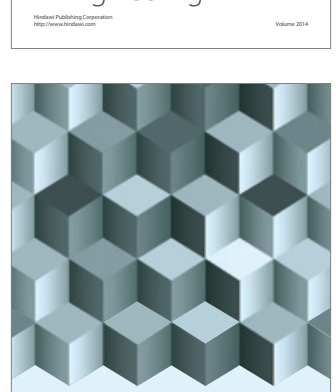

Journal of

Function Spaces
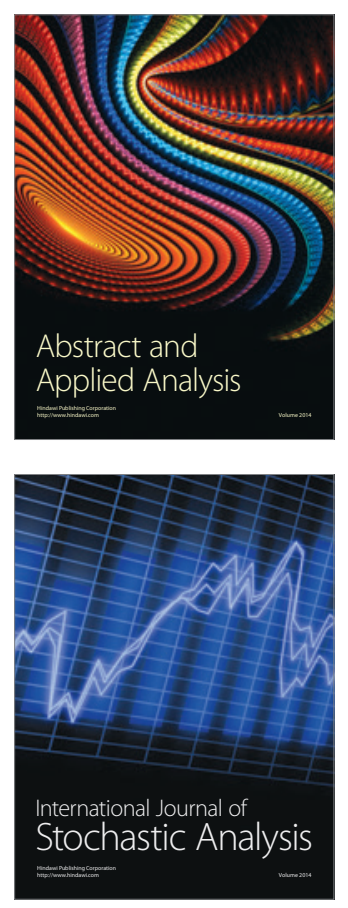

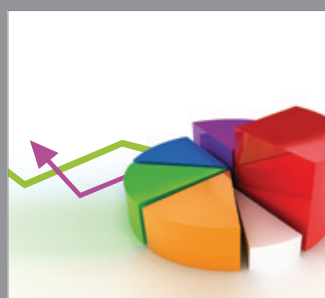

ournal of

Probability and Statistics

Promensencen
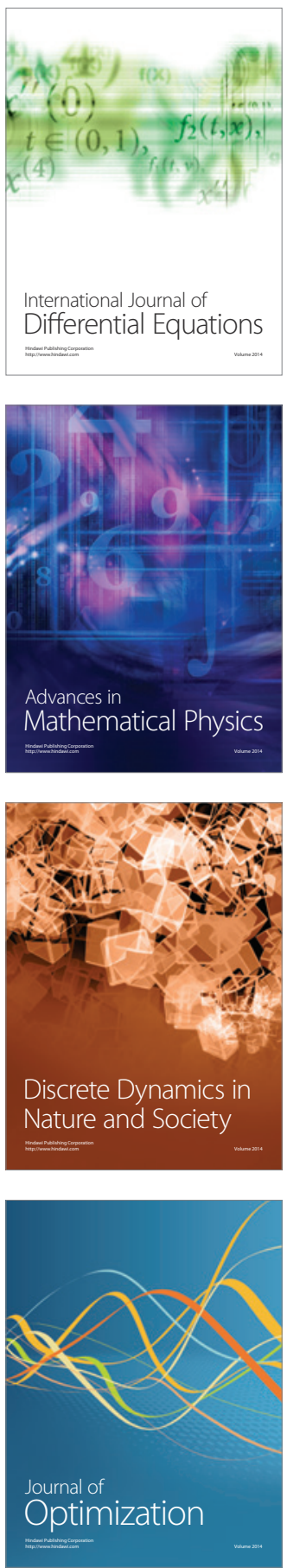